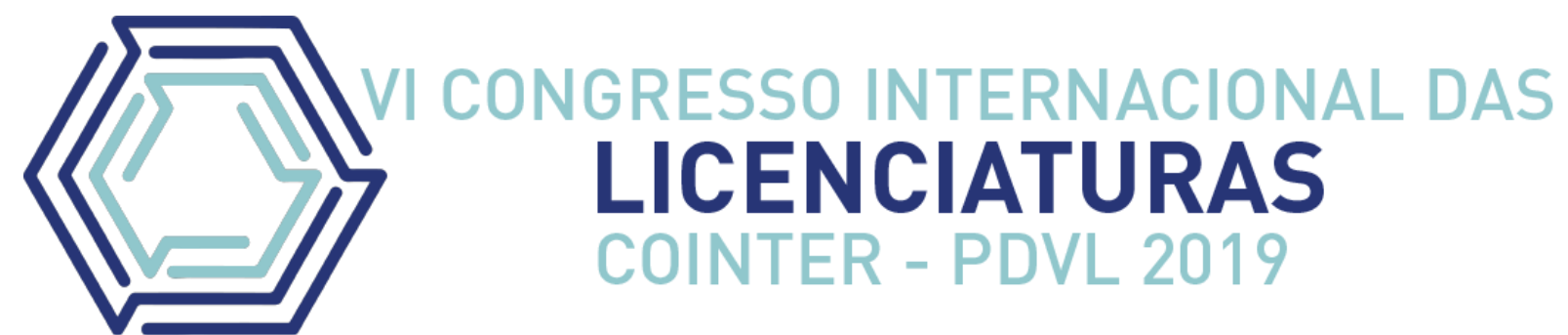

\title{
O PAPEL DA EDUCAÇÃo HUMANITÁRIA PARA O BEM ESTAR DE FELINOS DOMÉSTICOS NAS IMEDIAÇÕES DO IFRN-CNAT
}

\author{
EL PAPEL DE LA EDUCACIÓN HUMANITARIA PARA EL BIENESTAR DE LOS \\ FELINOS DOMÉSTICOS EN INMEDIACIONES IFRN-CNAT
}

\begin{abstract}
THE ROLE OF HUMANITARIAN EDUCATION FOR THE WELLNESS OF DOMESTIC FELINES IN IFRN-CNAT IMMEDIATIONS
\end{abstract}

\author{
Apresentação: Comunicação Oral \\ Carmem Sara Pinheiro de Oliveira ${ }^{1}$; Kivia Moreira Nunes²; Maria Gabriella Pinheiro de \\ Oliveira $^{3}$; Adriane Moreira Nunes ${ }^{4}$; Claudia Regia Gomes Tavares ${ }^{5}$
}

DOI: https://doi.org/10.31692/2358-9728.VICOINTERPDVL.2019.0094

\section{Resumo}

O bem estar animal é um termo relacionado ao estado físico e mental do animal, influenciando na saúde tanto do animal, quanto humana. Por sua vez, a Educação Humanitária é uma interessante ferramenta - tendo em vista que as diferentes culturas dos povos estão diretamente conectadas com a forma em que tratam a relação humano-animal - em que há a valorização do respeito e da compaixão para com os animais, pessoas e meio ambiente, sendo possível informar, mudar hábitos e transformar as pessoas em difusoras de conhecimento e em vigilantes ativos. Nessa perspectiva, a Educação Humanitária relaciona-se com a função da Educação Ambiental, uma vez que, esta é ferramenta para a preservação de espécies, de ecossistemas e de ambientes naturais. Assim, os objetivos desta pesquisa são compreender o nível de entendimento sobre as questões abordadas pela educação humanitária e incentivar a reflexão acerca do conceito desta educação, da posse responsável, do controle populacional e do bem estar dos gatos das imediações do IFRN/CNAT. Para isso, houve a aplicação de questionário online para 44 alunos do Ensino Técnico Integrado do Instituto Federal do RN. No quesito de quais cursos foram mais presentes no Questionário Investigativo, o Curso Técnico Integrado Controle Ambiental reflete 63,6\% dos entrevistados, enquanto o Curso de Mineração representa $20,5 \%$ do público alvo. Além deles, há Mecânica (9,1\%), Geologia (4,5\%) e Eletrotécnica $(2,3 \%)$. Diante dos resultados, percebeu-se a falta da Educação Humanitária, entrelaçada à

\footnotetext{
${ }^{1}$ Professora Mestra, Universidade Federal do Rio Grande do Norte, sarinhac.s@hotmail.com

2 Técnico Integrado em Controle Ambiental, Instituto Federal de Educação, Ciência e Tecnologia do Rio Grande do Norte, kiviamoreira9@gmail.com

${ }^{3}$ Nutrição, Universidade Potiguar, gabriella_gaby9@hotmail.com

4 Técnico Integrado em Mecânica, Instituto Federal de Educação, Ciência e Tecnologia do Rio Grande do Norte, adri.nunes1930@hotmail.com

5 Professora Doutora, Instituto Federal de Educação, Ciência e Tecnologia do Rio Grande do Norte, claudia.tavares@ifrn.edu.br
} 
Educação Ambiental, nos entrevistados. No ano de 2018, 41 gatos dentro do Instituto foram castrados, sendo 25 fêmeas e 16 machos. Em tal ano, houve cerca de quatro feiras de adoção, tendo 27 gatos adotados. Assim, propaga-se a ideia da posse responsável, levantada pelo público alvo ao 63,6\% afirmarem que não possuíam conhecimento acerca de casos envolvendo abandono animal em seu ambiente de convívio. Neste contexto, a Educação Humanitária no Instituto, se torna essencial, visando a intervenção da comunidade e da própria Instituição no conhecimento dos discentes a fim de mudar esse cenário. Portanto, é preciso que hajam cuidados visando a propagação desse bem estar. Logo, entendemos que a Educação Humanitária é uma interessante ferramenta, pois há a valorização do respeito e da compaixão para com os animais, pessoas e meio ambiente.

Palavras-Chave: Bem estar animal, Educação, Gatos, Saúde.

\section{Resumen}

El bienestar animal es un término relacionado con el estado físico y mental del animal, que influye en la salud de los animales y los humanos. A su vez, la educación humanitaria es una herramienta interesante, dado que las diferentes culturas de los pueblos están directamente relacionadas con la forma en que tratan la relación humano-animal, en la que se valora el respeto y la compasión por los animales, las personas y el medio ambiente, pudiendo informar, cambiar hábitos y transformar a las personas en difusores de conocimiento y vigilantes activos. En esta perspectiva, la Educación Humanitaria está relacionada con el papel de la Educación Ambiental, ya que es una herramienta para la preservación de especies, ecosistemas y ambientes naturales. Por lo tanto, los objetivos de esta investigación son comprender el nivel de comprensión de los problemas abordados por la educación humanitaria y fomentar la reflexión sobre el concepto de esta educación, la propiedad responsable, el control de la población y el bienestar de los gatos en las cercanías de IFRN/CNAT. Para esto, se aplicó un cuestionario en línea a 44 estudiantes de la Educación Técnica Integrada del Instituto Federal de RN. Con respecto a los cursos que estuvieron más presentes en el Cuestionario de investigación, el Control ambiental del curso técnico integrado refleja el $63.6 \%$ de los encuestados, mientras que el Curso de minería representa el $20.5 \%$ de la audiencia objetivo. Además de ellos, hay Mecánica $(9.1 \%)$, Geología (4.5\%) y Electrotecnia (2.3\%). Dados los resultados, se notó la falta de Educación Humanitaria, entrelazada con la Educación Ambiental, en los entrevistados. En 2018, se castraron 41 gatos dentro del Instituto, 25 hembras y 16 machos. En ese año, hubo alrededor de cuatro ferias de adopción, con 27 gatos adoptados. Por lo tanto, la idea de propiedad responsable, planteada por el público objetivo al 63,6\%, afirma que no tenía conocimiento sobre los casos de abandono de animales en su entorno de vida. En este contexto, la educación humanitaria en el Instituto se vuelve esencial, con el objetivo de la intervención de la comunidad y la propia institución en el conocimiento de los estudiantes para cambiar este escenario. Por lo tanto, se debe tener cuidado para propagar este bienestar. Por lo tanto, entendemos que la Educación Humanitaria es una herramienta interesante, ya que existe el aprecio por el respeto y la compasión por los animales, las personas y el medio ambiente.

Palabras Clave: Bienestar animal, Educación, Gatos, Salud.

\section{Abstract}


Animal welfare is a term related to the physical and mental state of the animal, influencing the health of both animal and human. In turn, humanitarian education is an interesting tool - given that the different cultures of peoples are directly connected with the way they treat the humananimal relationship - in which respect and compassion for animals are valued., people and the environment, being able to inform, change habits and transform people into knowledge diffusers and active vigilantes. In this perspective, Humanitarian Education is related to the role of Environmental Education, since it is a tool for the preservation of species, ecosystems and natural environments. Thus, the objectives of this research are to understand the level of understanding on the issues addressed by humanitarian education and encourage reflection on the concept of this education, responsible ownership, population control and welfare of cats in the vicinity of IFRN/CNAT. For this, an online questionnaire was applied to 44 students of the Integrated Technical Education of the Federal Institute of RN. Regarding which courses were most present in the Investigative Questionnaire, the Integrated Technical Course Environmental Control reflects $63.6 \%$ of respondents, while the Mining Course represents $20.5 \%$ of the target audience. Besides them, there are Mechanics (9.1\%), Geology (4.5\%) and Electrotechnics (2.3\%).Given the results, it was noticed the lack of Humanitarian Education, intertwined with Environmental Education, in the interviewees. In 2018, 41 cats inside the Institute were neutered, 25 females and 16 males. In that year, there were about four adoption fairs, with 27 adopted cats. Thus, propagates the idea of responsible ownership, raised by the target audience to $63.6 \%$ claim that they had no knowledge about cases involving animal abandonment in their living environment. In this context, humanitarian education in the Institute becomes essential, aiming at the intervention of the community and the institution itself in the knowledge of the students in order to change this scenario. Therefore, care must be taken to propagate this wellbeing. Therefore, we understand that Humanitarian Education is an interesting tool, as there is the appreciation of respect and compassion for animals, people and the environment.

Keywords: Animal Welfare, Education, Cats, Health.

\section{Introdução}

A relação humano-animal, assim afirma Santos et al. (2013), não é atual, principalmente quando se trata do homem e dos animais por ele domesticados, sendo essa relação afetada, diretamente, pelas diferentes culturas dos povos. Dessa maneira, a fim de que esse relacionamento seja cada vez mais saudável, é essencial que haja o conhecimento dos conceitos interligados a esse vínculo humano-animal, exemplificado: o bem estar animal.

O bem estar animal (BEA) é um termo empregado em diversas sociedades humanas (FREIRE et al., 2016), "podendo ser utilizado a todos os animais do planeta" (FARACO \& SEMINOTTI, 2004 apud CORSO et al., 2013). Segundo Paula (2010), a definição de bemestar animal está relacionada tanto ao físico, quanto ao emocional do animal, sendo possível analisar essa por meio de evidências comportamentais. 
Consoante a Broom e Molento (2004), o BEA influencia na saúde tanto animal, quanto humana, em vista do aumento do vínculo entre os homens e animais, sequenciando a "superpopulação de animais domésticos errantes, o aumento de maus-tratos aos animais e o aumento da transmissão das zoonoses nos nossos centros urbanos" (FREIRE et al., 2016). O conceito de zoonose é definido pela Organização Mundial de Saúde - OMS (2019) como uma doença ou infecção naturalmente transmissível de animais vertebrados para os seres humanos.

Tendo um conceito concreto, conforme Paula (2010), as “Cinco Liberdades" são utilizadas como princípios objetivados a alcançar o que se chama o Bem-estar animal. A Liberdade Nutricional refere-se ao animal livre de fome, sede e desnutrição, enquanto a Liberdade Psicológica está relacionada ao animal livre de medo e estresse. A Liberdade Sanitária interliga-se ao animal liberto da dor, doenças e ferimentos, ao passo que a Liberdade Ambiental diz sobre o animal livre para movimentar-se em ambientes adequados à sua espécie. E, por fim, a Liberdade Comportamental, que trata do animal permitido de expressar o comportamento natural de sua espécie.

Dessa forma, para a propagação dos conceitos das Cinco Liberdades, Almeida et al. (2014) afirma que a Educação Humanitária é uma interessante ferramenta - tendo em vista que as diferentes culturas dos povos estão diretamente conectadas com a forma em que tratam a relação humano-animal, como afirma Freire et al. (2016) - uma vez que, por meio dessa, há a valorização do respeito e da compaixão para com os animais, pessoas e meio ambiente, sendo possível informar, mudar hábitos e transformar as pessoas em difusoras de conhecimento e em vigilantes ativos, segundo Corso et al. (2013). Nessa perspectiva, a Educação Humanitária por sua vez, relaciona-se com a função da Educação Ambiental, uma vez que, de acordo com Martins e Nishijima (2010), esta é ferramenta para a preservação de espécies, de ecossistemas e de ambientes naturais.

As Instituições de Ensino, que apresentam problemas vinculados a esta temática, devem tomar para si a Responsabilidade Socioambiental (RSA) dessa população, objetivando a saúde e bem-estar da comunidade no convívio com esses animais e contribuindo no controle de zoonoses nessa interface homem-animal.

Por isso, é preciso que haja o ensino da educação humanitária, a fim de que exista o maior controle populacional desses animais e a diminuição do abandono tendo, consequentemente, a redução da propagação da zoonose e o aumento do bem-estar felino. Dado o aumento exponencial da população de felinos, oriundos do entorno do Instituto (Federal de 
Educação, Ciência e Tecnologia do Rio Grande do Norte, campus Natal-Central), explicitado por Galdino et al. (2017), a preocupação com a propagação de doenças no meio ambiente vem crescendo, tornando o tema relevante à sociedade. E, ressaltando Garcia (2005), a educação e a conscientização da pessoa responsável são alicerces de programas de controle de populações animais.

Dessa forma, os objetivos desta pesquisa são compreender o nível de entendimento sobre as questões abordadas pela educação humanitária, bem como incentivar a reflexão acerca do conceito desta educação, da posse responsável, do controle populacional e do bem estar dos gatos das imediações do IFRN/CNAT.

\section{Fundamentação Teórica}

Com a Educação Humanitária, Corso et al. (2013) enfatiza a inter-relação de todos os seres vivos, não somente sendo tema a ser investigado pelos estudantes e por outros sujeitos (educadores), desafiando-os a procurar soluções, investigando a fundo essas problemáticas evidenciadas pelo estudo sobre a Educação Humanitária, tornando-os críticos e conscientes da importância de seus próprios atos na transformação da sociedade.

Consoante Weil (2013), a base da Educação Humanitária é composta por quatro elementos fundamentais: fornecer informações exatas, a fim dos indivíduos entendam as consequências de suas decisões; incentivar os 3 Cs: Curiosidade, Criatividade e Crítica, assim, os sujeitos são capazes de avaliar informações e resolver problemas; propor os 3Rs: Reverência, Respeito e Responsabilidade, para que as pessoas possam agir com gentileza e integridade; e finalizando, oferecer escolhas positivas que beneficiem os próprios sujeitos, outros indivíduos, o meio ambiente e os animais.

De acordo com Freire et al. (2016), pensa-se na Educação Humanitária em Bem-estar animal como metodologia pedagógica com perspectiva na construção de valores e crenças positivas para com os animais e para com a sociedade e meio ambiente como um todo, havendo a melhora da saúde pública, o maior controle populacional de animais através da castração, sendo o método mais indicado para evitar a procriação (GALLANI et al., 2010), bem como auxilia a conceituar a chamada posse responsável, bem diz Carvalho et al. (2011). O conceito de posse responsável, segundo Santana et al. (2004), não remete somente ao cuidar com carinho e propiciar bem estar ao animal, mas também adotar as medidas para que ele cresça sadio e não transmita doenças ao homem. 
A posse animal, por Gallani et al. (2010), diz respeito a não apenas cuidar com carinho e conceber bem-estar ao animal, mas também medidas com a finalidade de que o animal cresça sadio e que não transmita doenças ao ser humano. A definição de Posse Responsável de animais de companhia, de acordo com Santana et al. (2004)

\footnotetext{
"É a condição na qual o guardião de um animal de companhia aceita e se compromete a assumir uma série de deveres centrados no atendimento das necessidades físicas, psicológicas e ambientais de seu animal, assim como prevenir os riscos (potencial de agressão, transmissão de doenças ou danos a terceiros) que seu animal possa causar à comunidade ou ao ambiente, como interpretado pela legislação vigente."
}

Sem a presença da consciência do papel de guardião do animal, tem por consequência o abandono do animal. Essa atitude de negligência sobre o animal contribui para a proliferação de zoonoses, uma vez que, assim afirma Silva e Victória (2011), gera o crescimento populacional, não existindo, portanto, um manejo adequado desses animais, surgindo doenças que podem ser transmitidas ao homem: raiva, leishmaniose, toxoplasmose, proliferação de parasitas; pulgas, carrapatos e sarna, agressões, acidentes de trânsito, poluição por dejetos, poluição sonora e outras perturbações.

Nessa visão, afirma Santos et al. (2013) que a posse responsável revela-se como um instrumento de amenização de problemas originados do aumento desordenado das populações de gatos, isto é, além da castração/esterilização, como solução da superpopulação de animais errantes, reduzindo os problemas da interação indesejada entre o homem e o animal (HOWE, 2006), há o controle populacional desses animais por meio da guarda animal, "sendo um valioso instrumento de Saúde Pública" (NOGUEIRA, 2009).

Isso é de extrema importância, pois como afirma Magnabosco (2006), o desequilíbrio na população animal acarretou em excessos populacionais e, juntando aos impasses da falta de saneamento e o crescimento desordenado das cidades, aumenta a possibilidade da propagação de zoonoses. Deve ser destacado que os aspectos do controle populacional, a posse responsável e assim como o bem estar animal, abordados pela Educação Humanitária, bem como pela Educação Ambiental, estão diretamente relacionados à população de gatos. Gallani et al. (2010) afirma que a atitude do abandono de gatos

“[...] contribui para a incidência das zoonoses, pois ocorre o crescimento populacional e o descuido com os animais. As condições de higiene, saúde e moradia desses animais 
não são adequadas e, por fim, o homem acaba sofrendo a consequência de seus próprios atos contraindo uma doença."

Além disso, Gomes (2009) corrobora que os interessados em conviver com os gatos assumem a responsabilidade de desenvolver e manter hábitos e posturas que promovam e preservem a saúde, o bem estar do animal e do meio ambiente. De acordo com Garcia (2005), a educação e a conscientização da pessoa responsável são os pilares de programas de controle de populações animais.

\section{Metodologia}

Como afirma Gil (1999), o questionário é uma técnica de investigação que tem por objetivo o levantamento de perspectivas sobre determinada realidade. Por isso, o trabalho objetivou o uso do Questionário, visando a verificação do nível de conhecimento acerca de temas que envolvem tanto a Educação Humanitária, quanto a Ambiental da comunidade do Instituto.

A fim de cumprir os objetivos deste artigo supracitados, houve a aplicação de um questionário online denominado Questionário Investigativo: Bem-estar Animal e Zoonoses (IFRN-Natal-Central), composto por quatorze perguntas fechadas.

Por meio dessas, foram abordados temas relacionados ao Bem-estar Animal, à Educação Humanitária, à posse responsável e à zoonose, associando-os ao contexto de vivência da população de gatos do Instituto Federal.

Para delimitar e atingir os fins deste trabalho, o questionário foi aplicado somente para os discentes de Cursos Técnicos Integrados do Instituto Federal do Rio Grande do Norte, campus Natal-Central. Nessa perspectiva, o questionário do tipo quantitativo alcançou cerca de 44 alunos, retirando a média dessas respostas por via estatística básica sob forma de percentual.

\section{Resultados e Discussão}

Em relação ao gênero com maior alcance entre os entrevistados, houve a maior presença do gênero feminino, compondo cerca de $75 \%$ dos entrevistados, enquanto o gênero masculino compõe $25 \%$ dos discentes alvos do questionário. Sobre a idade, $72,7 \%$ dos estudantes possuem de 16 a 18 anos. Vale ressaltar a presença de $25 \%$ das pessoas que possuem 18 anos ou mais que foram entrevistadas. 
Entre os dialogados, cerca de 43,2\% estão cursando o segundo ano do seu respectivo Curso Técnico Integrado. Entre as porcentagens da presença do terceiro e do quarto ano foram próximas: $27,3 \%$ compõem os indivíduos cursando o terceiro, enquanto $29,5 \%$ representam o quarto.

No requisito dos quais cursos foram mais presentes no Questionário Investigativo, o Curso Técnico Integrado Controle Ambiental reflete 63,6\% dos entrevistados, enquanto o Curso de Mineração representa 20,5\% do público alvo. Além deles, há Mecânica $(9,1 \%)$, Geologia (4,5\%) e Eletrotécnica (2,3\%). Abaixo, no Gráfico 1, tem-se os Cursos Técnicos Integrados dos alunos que foram entrevistados.

Gráfico 1. Percentual ilustrativo dos cursos Técnicos Integrados participantes da pesquisa.

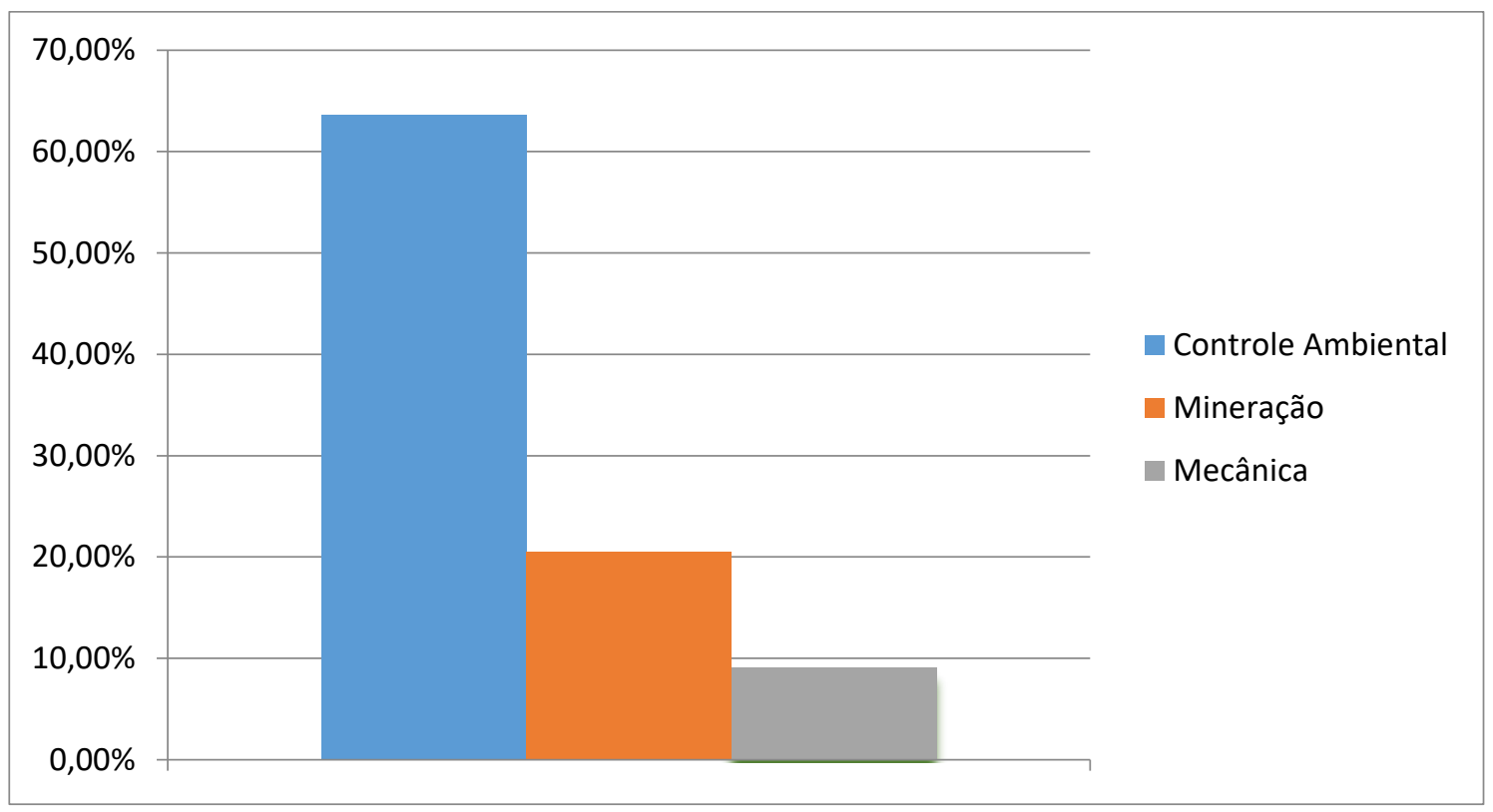

Fonte: Autoria própria (2019).

Após essas perguntas, houveram três questões essenciais para este Questionário: sobre Educação Humanitária, Bem-estar animal e Zoonose, respectivamente. Nos gráficos a seguir (2, 3 e 4), demonstramos os resultados.

Gráfico 2. Questionamento acerca do conhecimento sobre "Educação Humanitária". 


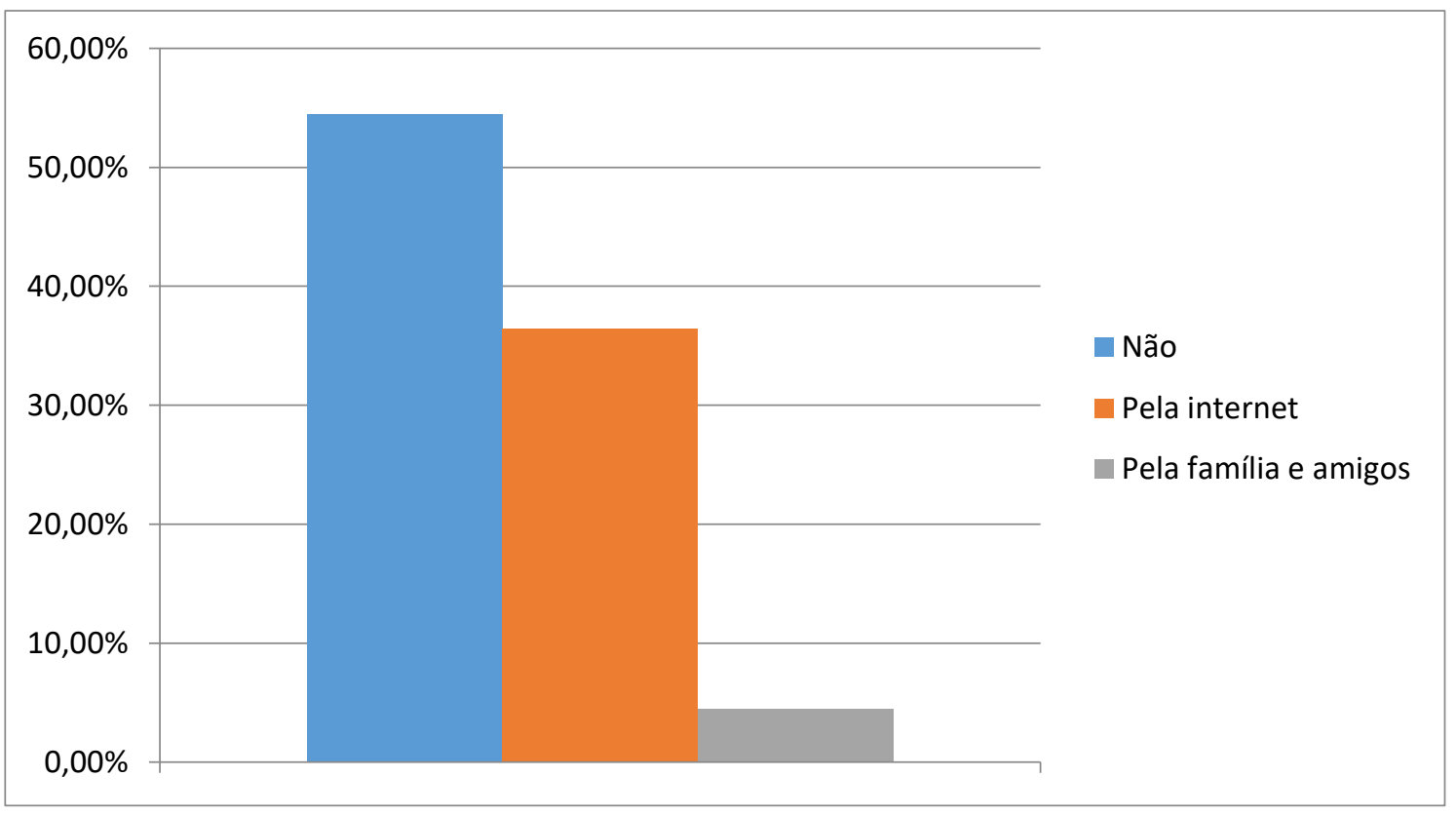

Fonte: Autoria própria (2019).

No Gráfico 2, que diz respeito as respostas acerca do conhecimento da existência da Educação Humanitária, temos que cerca de 54,5\% dos entrevistados nunca ouviram falar do conceito anteriormente a esse Questionário. Por outro lado, 36,4\% já tiveram contato com a Educação Humanitária pela internet e $4,5 \%$ por amigos. É importante ressaltar que somente 2,3\% dos entrevistados souberam da Educação Humanitária pelo Instituto Federal do Rio Grande do Norte. Esse dado, bem como a das duas perguntas que se seguirão, refletem a necessidade de uma maior inserção desses temas em disciplinas no Instituto.

Gráfico 3. Questionamento acerca do conhecimento sobre "Bem-estar Animal". 


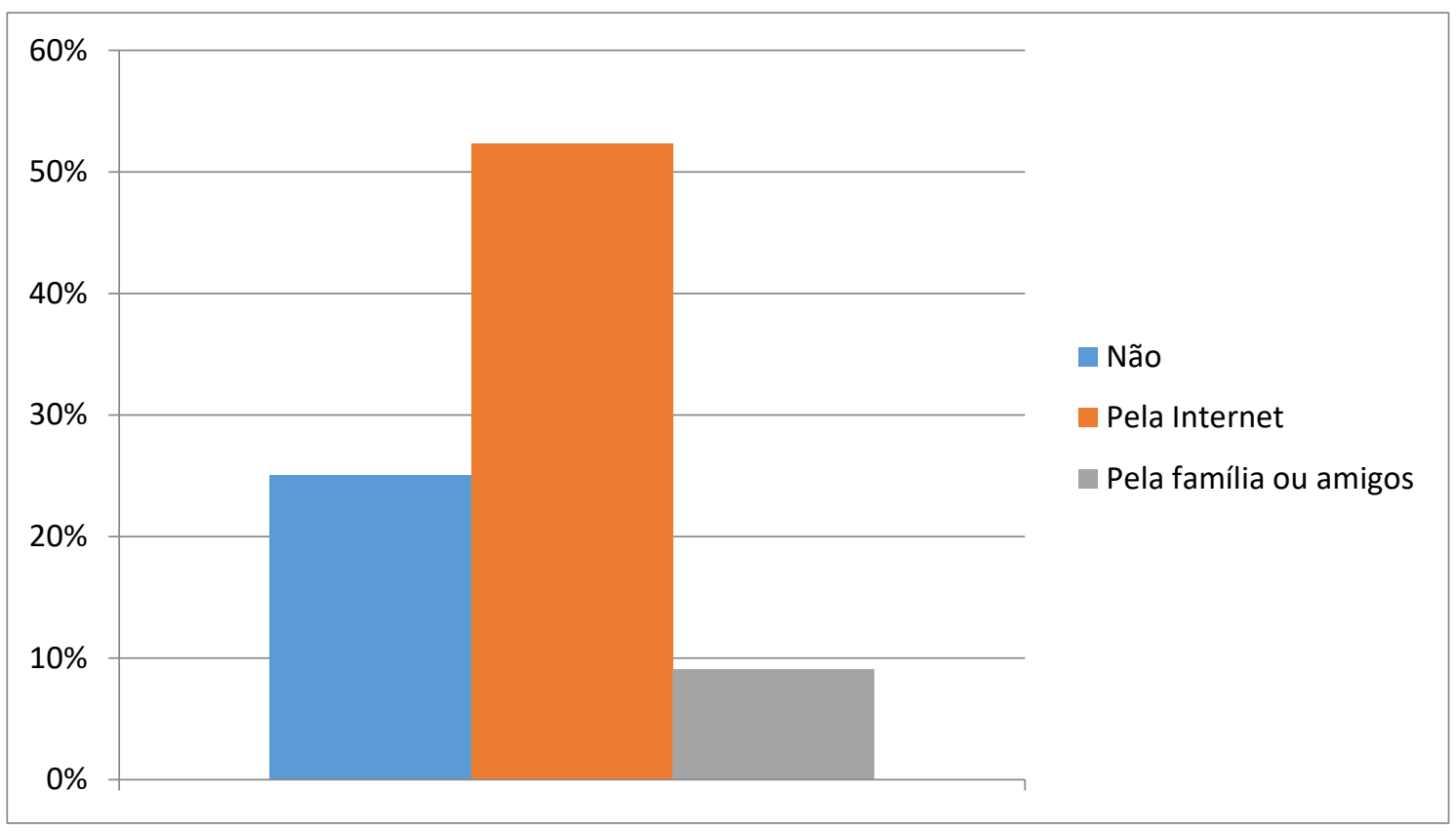

Fonte: Autoria própria (2019).

A partir do Gráfico 3, percebe-se que há um maior entendimento sobre o Bem-estar Animal pelos estudantes do IFRN-CNAT. Em torno de 52,3\% do público alvo conhecem o conceito por meio da internet e 9,1\% pela família ou amigos. Porém, $25 \%$ não têm a ideia do BEA. Ainda há 6,8\% das pessoas que ouviram falar do termo a partir dos professores do Instituto Federal e outros 6,8\% que sabem dessa ideia através da televisão.

Gráfico 4. Questionamento acerca do conhecimento sobre "Zoonose”. 


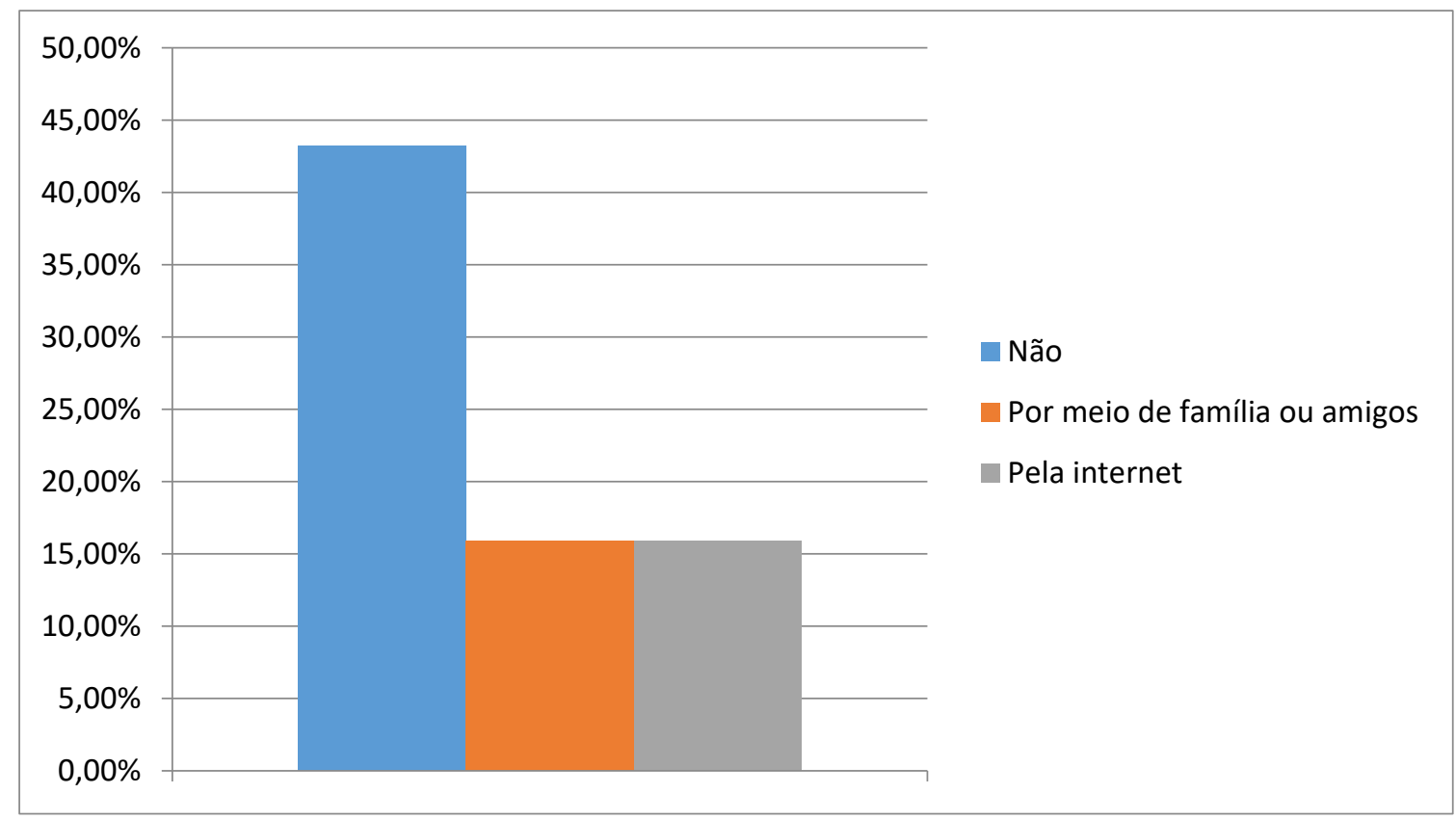

Fonte: Autoria própria (2019).

A partir da questão sobre Zoonose, explicitada pelo Gráfico 4, percebe-se que 43,2\% dos indivíduos não têm o conhecimento sobre o assunto. Apesar disso, 15,9\% entendem do conceito através da família ou amigos, bem como os outros $15,9 \%$ possuem a ideia a partir da internet. É essencial afirmar que somente $9,1 \%$ do público entrevistado conheceram o conceito pelo Instituto.

Enfatizado pelas três perguntas citadas acima, é perceptível a ausência da Educação Humanitária, entrelaçada à Educação Ambiental, nos entrevistados. Em todas as questões, a presença do Instituto foi mínima. Por conseguinte, isso acarreta nos problemas mencionados em relação à população de gatos no IFRN-CNAT, isto é, há o aumento dessa comunidade, não tendo o Instituto a consciência enquanto a isso, e, dessa forma, a própria comunidade não possui a educação necessária para lidar com essa situação.

Consoante a Silva e Victória (2011), a falta da Educação Humanitária, junto à Ambiental, sequencia no aumento da propagação da Zoonose, bem como a extensão dessa população felina, prejudicando o meio ambiente e a saúde pública. Por conta disso, 95,5\% dos entrevistados acham necessária a existência desses temas que compõem a Educação Humanitária na ementa de suas disciplinas ou trabalha-las melhor ao longo do ano letivo.

Através dessa perspectiva, compreende-se a necessidade de, além da melhor aplicabilidade diante dessas questões na ementa das disciplinas dos discentes, projetos e programas relacionados a esses temas dentro do Instituto, posto que essa relevância deriva 
desses poderem intervir na formação de uma sociedade, podendo reeducar os sentidos, como afirma Mello (2013).

Dessa maneira, 97,7\% dos alunos acreditam ser importante a presença de programas e projetos relacionados aos cuidados e ao controle populacional desses animais no IFRN-CNAT. Com isso, mostra-se a relevância do Projeto de Extensão: Controle populacional de gatos nas imediações do IFRN/CNAT, ativo desde 2017, que, já afirmado por Galdino et al. (2017), devido à sua grande importância sociocultural e econômica, considerando-se que todos os custeios do projeto se darão pela arrecadação dos recursos e serviços para proporcionar a segurança e saúde desses felinos do IFRN/CNAT e, por fim, garantir a segurança, saúde e a educação da comunidade presente neste ambiente.

No ano de 2018, 41 gatos dentro do Instituto foram castrados, sendo 25 fêmeas e 16 machos. Em tal ano, houve cerca de quatro feiras de adoção, tendo 27 gatos adotados. Assim, propaga-se a ideia da posse responsável, levantada pelo público alvo ao 63,6\% afirmarem que não possuíam conhecimento acerca de casos envolvendo abandono animal em seu ambiente de convívio, em que Santana et al. (2004) conceitua, levando à ideia de não somente existe o cuidado e o carinho em torno da adoção de um gato, mas sim prevenir que esse possa causar prejuízo à sociedade ou ao meio ambiente.

\section{Conclusões}

É preciso compreender que a situação da população de gatos no IFRN-CNAT deve-se, principalmente, à falta da abordagem da Educação Humanitária, interligada à Educação Ambiental, dentro do Instituto, gerando o aumento dessa população. As abordagens associadas à Educação Humanitária escolhidas para compreender o nível de conhecimento sobre a questão da população felina dos educandos do IFRN-CNAT foram a Zoonose, Bem-estar Animal, posse responsável e a própria questão da Educação Humanitária.

Diante das respostas dos entrevistados, percebe-se a falta da Educação Humanitária no Instituto, sendo essencial a intervenção da comunidade e da própria Instituição a fim de mudar esse cenário. Para isso, necessita-se de projetos e programas que passem a ideia da Educação Humanitária: a valorização do respeito e da compaixão para com os animais, pessoas e meio ambiente.

\section{Referências}


ALMEIDA, J. F. Educação humanitária para o bem-estar de animais de companhia. ENCICLOPÉDIA BIOSFERA, Centro Científico Conhecer - Goiânia, v.10, n.18, 2014.

BROOM, D. M.; MOLENTO, C. F. M. Bem-estar animal: conceito e questões relacionadas Revisão. Archives of Veterinary Science, v.9, n.2, 2004.

CARVAlHO, A. A. B.; GRISÓLIO, A. P. R.; BUENO, G. M.; TESTI, A. J. P.; MARTINS, M. C.; PORTELA, L. C.; SERVIDONE, J. S.; NUNES, J. O. R. Caracterização da população de cães e gatos e avaliação do nível de conhecimento dos moradores sobre zoonoses e posse responsável de animais de estimação, em bairros do município de Jaboticabal/SP. Rev. Ciênc. Ext., v.7, n.2, 2011.

CORSO, A. S.; KONZEN, C; M. T.; MARCON, D. F.; Santos JUNIOR, N. L.; MELLO, D. M. S. EDUCAÇÃO HUMANITÁRIA PARA O BEM-ESTAR ANIMAL: PERCEPÇÃO E ATITUDES DOS ALUNOS DO ENSINO FUNDAMENTAL DO MUNICÍPIO DE REALEZA - PR. In: Congresso Brasileiro da ANCLIVEPA. 34., 2013, Natal. Anais eletrônicos.... Natal: Edufersa, 2013.

FRASER, D. Animal ethics and animal welfare science: bridging the two cultures. Applied Animal Behaviour Science, v. 65, n. 3, 1999.

FREIRE, R.; MELO, C. O.; CAETANO, W.; LACCHIA, A. P. EDUCAÇÃO HUMANITÁRIA NA SENSIBILIZAÇÃO PARA O BEM-ESTAR ANIMAL E NA IMPLEMENTAÇÃO DESTA TEMÁTICA NO CURRÍCULO DO ENSINO BÁSICO DE CAMPINA GRANDE, PB. In: Congresso Nacional de Educação. 3., 2016, Campina Grande. Anais eletrônicos... Campina Grande: Editora Realize, 2016.

GALDINO, A. P. M. JESUS, J. R. N.; SILVA, I. M. F.; AZEVEDO, L. F. S.; TAVARES, C. R. G. Controle populacional de gatos no IFRN: estratégia para a prevenção de riscos biológicos. In: Semana de Ciência, Tecnologia e Extensão do IFRN. 3., 2017, Natal. Anais eletrônicos... Natal: PROEX, nov. 2017.

GALLANI, S. U.; QUEIROZ, L. H.; VALLADÃO, G. M. R.; RODRIGUES, T. O.; PIRES, M. M.; PIRES, M. C. Conceitos e práticas de posse responsável e controle populacional de cães e gatos dos moradores de bairros próximos ao campus do curso de medicina veterinária da UNESP - Araçatuba. São Paulo, 2010. Disponível em: <http://www.conhecer.org.br/download/GUARDA\%20RESPONSAVEL/Leitura\%20anexa\% 206.pdf>. Acesso em: 31 jan. 2019.

GARCIA, R. C. Controle de populações de cães e gatos em área urbana: uma experiência inovadora na Grande São Paulo. Saúde Coletiva, São Paulo, v. 5, n. 2, 2005.

GIL, A. C. Métodos e técnicas de pesquisa social. São Paulo: Atlas, 1999.

GOMES, L. H. Programa de controle de Populações de cães e gatos do estado de São Paulo. Suplemento 7 do Boletim Epidemiológico Paulista, v. 6, 2009. Disponível em: <http://pt.scribd.com/doc/40375961/bepa-suple7v6-caesgatos>. Acesso em: 31 jan. 2019. 
HOWE, L. M. Surgical methods of contraception and sterilization. Theriogenology, v. 66, n. 3, 2006.

MAGNABOSCO, C. População domiciliada de cães e gatos em São Paulo: perfil obtido através de um inquérito domiciliar multicêntrico. 2006. Dissertação (Mestrado em Epidemologia) - Universidade de São Paulo. São Paulo, p. 110, 2006.

MARTINS, L. A. R.; NISHIJIMA, T. Preservação ambiental e qualidade de vida em comunidades quilombolas, Revista Eletrônica em Gestão, Educação e Tecnologia Ambiental REGET-CT/UFSM, v.1, n. 1, 2010.

MELLO, A. S. Lazer e educação ambiental: relato de experiências na formação inicial em educação física. Licere, Belo Horizonte, v.16, n.2, 2013.

NOGUEIRA, F. T. A. Posse responsável de animais de estimação no Bairro da graúna - Paraty, RJ. Revista Educação Ambiental BE-597, v. 2, 2009.

OMS - Organização Mundial da Saúde. Zoonoses. Disponível em: <http://www.who.int/topics/zoonoses/en/>. Acesso em 24 fev. 2019.

PAULA, P. M. C. Estratégias adicionais no controle populacional de cães de rua. 2010. Dissertação (Mestrado em Ciências Veterinárias) - Universidade Federal do Paraná. Curitiba, p. 72, 2010.

SANTANA, L. R.; MACGREGOR, E.; SOUZA, M. F. A.; OLIVEIRA, T. P. Posse responsável e dignidade dos animais. In: Congresso Internacional de Direito Ambiental. 8., 2004, São Paulo. Anais eletrônicos... São Paulo: Instituto O Direito por um Planeta Verde, 2004.

SANTOS, E. M. S.; SANTOS, H. O.; SANTOS, R. A.; ROCHA, M. H. F. F.; SILVA, C. P.; MACEDO-SOBRINHO, J. F. EDUCAÇÃO AMBIENTAL E POSSE RESPONSÁVEL DE ANIMAIS DOMÉSTICOS NO COMBATE À LEISHMANIOSE NO MUNICÍPIO DE ARAÇUAÍ, MG. Revista Cidadania em Ação, Florianópolis, v. 7, n. 1, 2013.

SILVA, D. T.; VICTÓRIA, C. AVALIAÇÃO DO NÍVEL DE CONHECIMENTO E ACEITAÇÃO DA CASTRAÇÃO E LEI DE POSSE RESPONSÁVEL NO MUNICÍPIO DE GARÇA/SP. Revista Científica Eletrônica de Medicina Veterinária, n. 17, 2011.

WEIL, Z. O poder e a Promessa da Educação Humanitária. 1. ed. São Paulo: Instituto Nina Rosa, 2013. 